\title{
STUDY ON TECHNOLOGY FOR PRECISION CORRECTION OF LAND SURVEY DATA BASEED ON PLOTTING RESULT DATA AND FEATURE POINTS
}

\author{
Guanghong Zeng ${ }^{\text {a }}$, Senhui Xie ${ }^{\text {b,* }}$, Jian Peng ${ }^{\text {b }}$, Mingxiu Liu ${ }^{\text {b }}$ \\ ${ }^{a}$ Guangzhou Municipal Land Resources and Housing Administrative Bureau, Guangzhou ,510030, China - \\ zgh0735@163.com \\ ${ }^{\mathrm{b}}$ Guangzhou hi-way Space Information System Technology Co., LTD, Guangzhou, 510030, China - \\ sameface@126.com, peision@sina.com, liumingxiu@21cn.com
}

KEY WORDS: Land Use, Correction, Model, Orthoimage, Mapping, Accuracy, Geometric, High resolution

ABSTRACT: High-precision land survey data is important foundation for fine management of land resources and enhancement of land use efficiency. The main method for land survey is remote sensing (RS) survey. However, the accuracy of data gained through this method is susceptible to image map, boundary interpretation, plotting process and other factors. To improve the accuracy, the method of precision correction of land investigation data that is based on high resolution image data with geometric correction model was discussed in detail. Technical test was conducted in some area of GuangZhou in China , and experimental results showed that the method can improve the accuracy of land survey data effectively.

Fund: Projects in the National Science \& Technology Pillar Program during the Twelfth Five-year Plan Period (No: 2012BAJ23B00)

\section{INTRODUCTION}

Land survey data is having a wide range of applications, and has been applied in every aspect of society, effecting and changing people's daily life ${ }^{[1]}$. In China, land survey based on remote sensing (RS) data is the main way to get the land use data. Surveyors interpret land use plots on high resolution RS images referring to historical materials, verify and revise the plots by field survey, and build database based on actual land-use status ${ }^{[2]}$. However, the accurate status of the land use data gained by this method is susceptible to several factors. The first is the accuracy of remote sensing data, as all the boundary line of plot is mapped from remote sensing data; the second is the method of boundary interpretation, different surveyor would get different boundary for the same plots as there are allowance, including visual error, pixel error and so on; the third is the tools used for plotting, generally GIS software is used for data processing, and it will generate errors during the process of coordinate transformation, file format conversion, scaling etc. These factors make quality problems of the data inevitable. Luckily, certain precision correction technology can help mitigate the defect.

In recent years, there are many papers discussed the accuracy of land survey data, and proposed some effective solutions to improve it. Overall, these solutions can be divided into two categories, the first is using precision-corrected high-resolution remote sensing image data for plot boundary 
interpretation to reduce the plotting error. For example, in china, Xie Zhi-ru ${ }^{[3]}$ analyzed the impact of relief on correction of remote sensing image and proposed to rectify the error resulted from altitude difference by orthographic correction method bringing in elevation data ; Zhao Jian-hui ${ }^{[4]}$ proposed to introduce DEM, RPC parameters and LPS modules to perform ortho rectification and LAN adjustment on QuickBird image ,which will later be used as base map in land survey . Moreover, G. B. GROOM ${ }^{[5]}$ has developed a range of post classification procedures to correct contextual errors associated with the use of spectral classification algorithms to improve land cover mapping from remotely sensed images ; N.G. Kardoulas ${ }^{[6]}$ investigated the geometric correction of Landsat MSS (80-metre pixel), Landsat TM (30-metre pixel), and SPOT Panchromatic (1 0-metre pixel) satellite images by using $1: 100,000$-scale topographic maps and GPS data in an area of Nigeria with the geometric corrections of first-degree polynomials; A. J. De Leeuw ${ }^{[7]}$ dealt with the composition of a correction function using ground control points and orthogonal functions; El-Manadili ${ }^{[8]}$ studied the ortho-rectification process for remote sensing image data as well, they proposed a Direct Linear Transformation Model for precision rectification of SPOT Imagery .

The second is focusing on the process of field survey and mapping in office operation, based on surveyors' land survey experience. For example, Xu Yu-Ying ${ }^{[9]}$ put forward some proposal and quality control requirements on digitization in office operation of land survey; Xu Hai-Jun ${ }^{[10]}$ introduced some quality control methods in charting base map , field survey, plotting in office operation and inserting database; Su Hao-jiang ${ }^{[11]}$ found out the precision rule and reason of effecting precision of data classified by terrain elements in 1:500 digital aerophotogrammetry mapping, then adopted measures in field mapping to warrant the precision quality of final map; Fan Ming-hua ${ }^{[12]}$ put forward the methods of debris erase and debris dissolve to deal with the problem of lots of debris when overly farmland map and the second land survey data .

These methods mentioned above achieved the purpose of improving the accuracy of land survey data to some extent, but they only reduced certain type of error in land survey data and aimed at part of the whole correction process, and the methods for reducing all types of plots errors were not proposed.

To make the survey data closer to physical truth, a method that could reduce all types of errors of land survey data through feature points and correction model which is discussed in this paper. Some area of GuangZhou in China was selected as a case to test the technology. The following section articulates the theory of the geometric correction model and the technology route of applying it to the correction of land survey data. The third part demonstrates the result of empirical study, taking some area of GuangZhou in China as an example. In the last section, summary about the method is concluded, some unsolved problems and proposals are discussed as well.

\section{THEORY OF GEOMETRIC CORRECTION MODEL}

\subsection{Source of Theory}

Geometry rectification of remote sensing imagery is using geometric correction model to approximate the geometric distortion of remote sensing image, which builds geometric distortion model by using remote sensing image and control points of 
standard map, and then apply this model for geometric correction, not considering the cause of the distortion ${ }^{[13]}$. In other words, geometry rectification of remote sensing imagery calculates the exact coordinate of all land patches, eliminates the errors in the coordinates of land patches, and therefore erases geometric distortion of the whole image. Considering the principle represented above, the reasons for geometry rectification of remote sensing imagery model can be applied for the correction of land survey data are stated.

\subsection{Geometric correction model}

The general mathematical expression of polynomial correction model is stated as follows:

$$
\left\{\begin{array}{l}
X=\sum_{i=0}^{N} \sum_{j=0}^{N-i} a_{i j} x^{i} y^{j} \\
Y=\sum_{i=0}^{N} \sum_{j=0}^{N-i} b_{i j} X^{i} y^{j}
\end{array}\right.
$$

In the above calculation formula, $X, Y$ represent feature node' coordinates of corrected area; $\mathrm{X}$, $\mathrm{y}$ corresponding to the $\mathrm{X}, \mathrm{Y}$, are the coordinates of the node whose data accuracy were not corrected; $a_{i j}, b_{i j}$ are undetermined coefficients of polynomial; $N$ is the number of polynomial, the value of which is determined by the character and type of the errors. The land survey data only need the plane coordinates ( $x, y$ coordinates), so the $N$ value should be 4 , namely 4 pairs of coordinates of corresponding feature points can solve the 8 undetermined coefficients. The land survey data polynomial correction model can be simplified as the following formula:

$$
\left\{\begin{array}{l}
X=a_{0}+a_{1} x+a_{2} y+a_{3} x y \\
Y=b_{0}+b_{1} x+b_{2} y+b_{3} x y
\end{array}\right.
$$

The way to solve the model coefficients is using the least square method combined with coordinates of 4 feature points.

\section{TECHNICAL ROUTE}

The main process of correcting the land survey data is using feature point coordinates $(x, y)$ to calculate the parameters of polynomial correction model, calculating the precise coordinates of every node, and rebuilding all features in the area. The precision correction includes 4 steps, they are Data preparation, Scope division, Nodes' coordinates correction and Output. The specified steps are stated as follows:

\subsection{Data preparation}

The process of land survey data's precision correction is using the coordinates of the feature point pairs as input data to solve the model's parameters, based on the land survey data that has accident errors. The data that should be prepared before applying the model to correction is shown in table 1.

\begin{tabular}{|l|l|}
\hline \multicolumn{1}{|c|}{ Data Name } & \multicolumn{1}{|c|}{ Application } \\
\hline $\begin{array}{l}\text { Land survey } \\
\text { data }\end{array}$ & $\begin{array}{l}\text { (1)Used as reference data of } \\
\text { feature point positioning } \\
\text { (2) Used as original data for } \\
\text { correcting land feature node's } \\
\text { coordinate }\end{array}$ \\
\hline $\begin{array}{l}\text { Feature points } \\
\text { data plotted }\end{array}$ & $\begin{array}{l}\text { Used as input data to solve the } \\
\text { model's parameters }\end{array}$ \\
\hline $\begin{array}{l}\text { Feature points } \\
\text { data } \\
\text { measured }\end{array}$ & $\begin{array}{l}\text { Used as input data to solve the } \\
\text { model's parameters }\end{array}$ \\
\hline DOM data & $\begin{array}{l}\text { (1)Used as reference data of } \\
\text { feature point positioning } \\
\text { (2) Used to check the result of the } \\
\text { correct data accuracy }\end{array}$ \\
\hline DEM data & $\begin{array}{l}\text { Used to analyze the change of } \\
\text { terrain }\end{array}$ \\
\hline
\end{tabular}

Table1: data preparation content summary table

Land survey data comes from interpreting DOM data, verifying and revising by field survey, and will be used as the original data for correcting.

In order to build feature point pairs, feature points data measured and feature points data plotted should be one 
correspondence. The identification of feature points is based on DOM data and land survey data.

\subsection{Area division}

To eliminate land survey data's plane errors and make land patches' coordinates approach to measurement data's coordinates, computational domain was recommended to be divided. It's divided from big rang to small rang. Firstly, the measurement area will be divided to a number of partitions for selecting feature points according to terrain change. Secondly, the partition for feature points' selection will be further divided for correction model to calculate. Partition for selecting feature points can make every partition's relief be as flat as possible, while the partition for correction model to calculate ensure the accuracy of solving model parameters. The technology and method for the division of measurement area is stated as follows:

(1) Dividing area for feature points' selection

Dividing an area for feature points' selection is based on topography change, and continuous area of close elevation will be divided to one area. According to the experiment, in order to ensure the flatness of one zone, the partition should meet the conditions stated below:

(1)Elevation difference must be smaller than $6 \mathrm{~m}$.

(2)Slope must be less than 5 degrees.

(3)There are more than 5 outstanding points in the image data.

(2) Dividing area for model

The standard for dividing areas for correction model to calculate is continuous area with 5 adjacent feature points. The method of dividing area for model includes 5 steps, they are stated as follows (figure 2):

(1)To construct Thiessen polygons based on the feature points in the individual area for feature points' selection;

(2)To calculate the number of adjacent Tyson Polygons of each Tyson Polygon in the individual area for feature selection;

(3)To group the Tyson Polygons on the rule of five feature points as a group and begin with the one with the least number of adjacent Tyson Polygons in the individual area for feature points' selection. Every Tyson Polygons should be grouped only once;

(4) Repeat step(3), until all the Tyson Polygons are grouped;

Output result, and each grouped polygons' coverage area is a scope for correction model to calculate.

\subsection{Node Coordinates correction}

Solving the model's coefficients accurately is the key of node coordinates' correction. To achieve the goal, the optimization program of the selection of feature points has been proposed. The process of realizing the program is stated as follows (as shown in Figure1):

(1) Choose four feature points of the same group, and use the coordinates of the four feature points to solve the coefficients;

(2) Calculate out the corrected coordinates of the fifth feature point;

(3) Calculate out the distance between the control point for rectification and the measurement point matching to the fifth feature point.

(4) Choose the other four feature points and repeat the step 2) and step 3 ).

(5) Choose the coefficients with the least distance that is calculated out in step 3);

(6) Calculate out the corrected coordinates of other patches' notes that are in the same scope with the coefficients. 


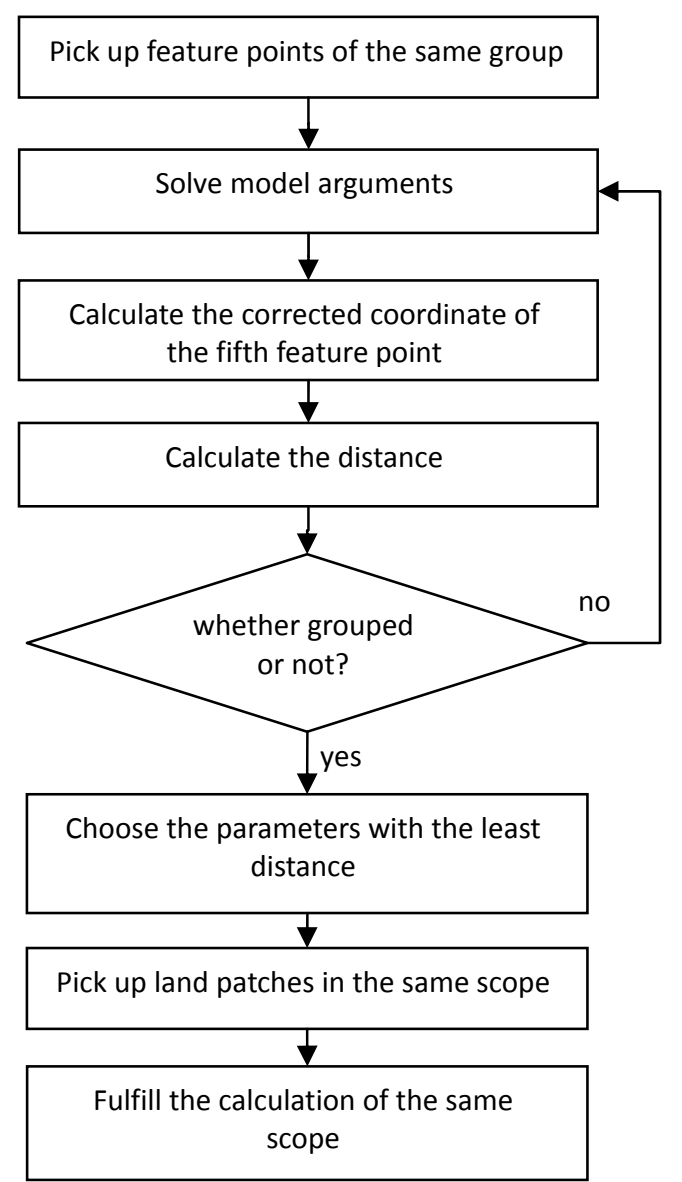

Figure1 flow chart of correction

\subsection{Output}

The process of output includes two-part work: feature reconstructing and results checking. Feature reconstructing is using corrected coordinates to construct the feature with reference to the original feature structure. Results checking include: checking out the position of the node and the area of the reconstructed polygon according to the standards as follows:

(1)The distance between the original position and the corrected position is less than the $1 / 5$ of the length of the feature boundary the node is on;

(2)The difference of polygon area between before and after reconstruction must be less than the $1 / 10$ of the area of the original polygon;

If the correcting results are unreasonable, the following approach should be taken:

(1)Exclude unreasonable feature points which the distance between the original position and the corrected position is great.

(2) Add new feature points in the appropriate place or re-select the feature points.

(3) Recalculate the corrective coordinates.

\section{EXPERIMENTAL RESEARCH}

Regional land survey data in Guangzhou is used to illustrate the technology for precision correction of land survey data, with the expectation of corrected coordinates close to the measurement coordinates.

The data used in this experiment is from the second land survey data of Guangzhou City, the data content is shown as follows:

\begin{tabular}{|l|l|}
\hline $\begin{array}{c}\text { Data } \\
\text { Name }\end{array}$ & \multicolumn{1}{|c|}{ data specification } \\
\hline $\begin{array}{l}\text { Land } \\
\text { use } \\
\text { data }\end{array}$ & $\begin{array}{l}\text { (1)Including the land use type } \\
\text { feature of cultivated land, garden, } \\
\text { forest land and so on. } \\
\text { (2) No gap between the polygons. }\end{array}$ \\
\hline $\begin{array}{l}\text { DOM } \\
\text { data }\end{array}$ & $\begin{array}{l}\text { (1) QuickBird image data, resolution } \\
\text { is } 0.5 \mathrm{~m} \text {, covering an area of } 80 \\
\text { square kilometers. } \\
\text { (2) 1:2000 standard map sheet. }\end{array}$ \\
\hline $\begin{array}{l}\text { Dem } \\
\text { Data }\end{array}$ & (1)The resolution is $0.5 \mathrm{~m}$ \\
\hline
\end{tabular}

Table2: Experimental raw data summary table

The process of empirical research is shown as follows:

(1) Pick up 680 feature points (as shown in Figure2) based on land use data and image. 


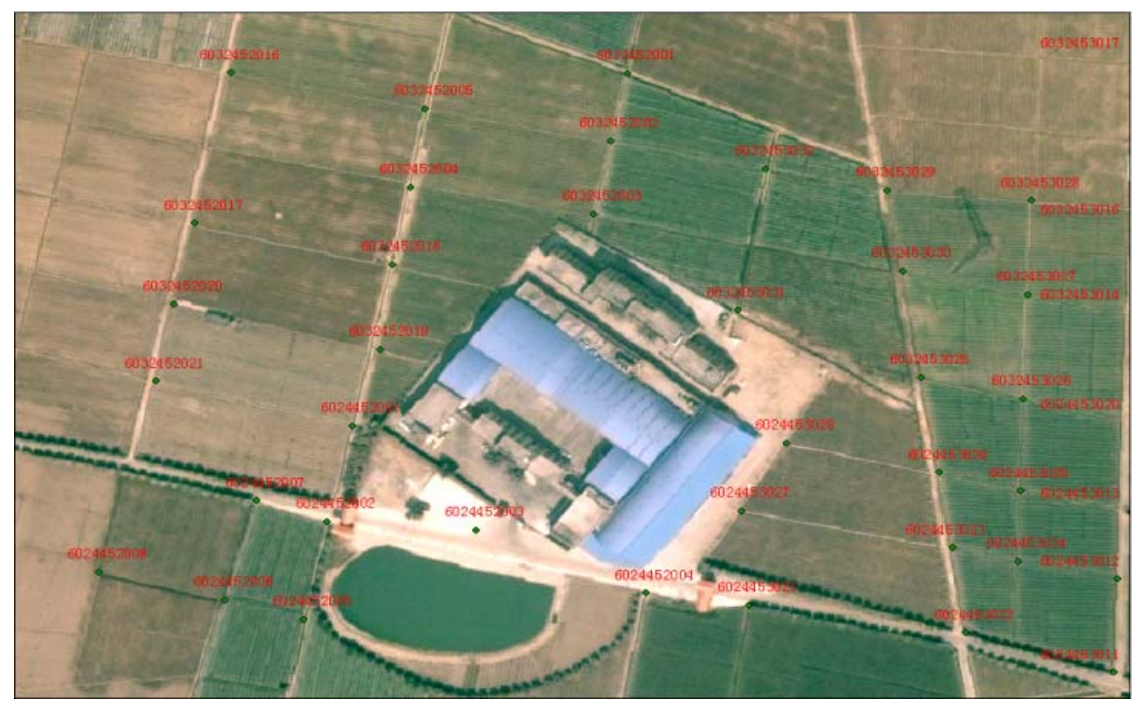

Figure 2: part of the selected feature points in experimental zone

(2)Collect the coordinates of feature points by high-precision GPS receivers with RTK mode.

(3) Correct the land-use feature nodes' coordinates with the land survey data precision correction model and reconstruct the polygons(as shown in Figure3).

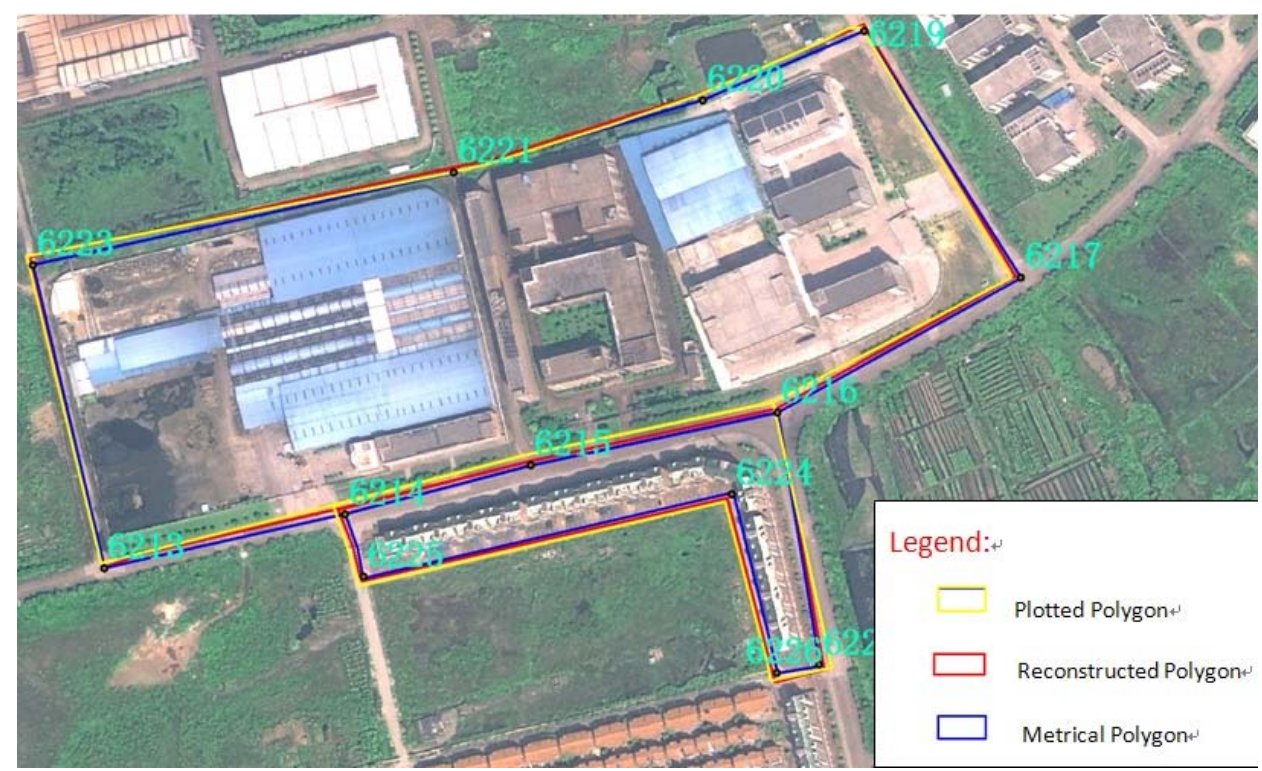

Figure3 The plotted polygons were reconstructed

(4) In order to check the accuracy of corrected coordinates, 8 notes were selected from 3 polygons randomly. The differences between the measurement coordinates and the corrected coordinates are shown in the table2.

It can be perceived from the table above, counting the distance between plotting point and the corresponding measuring point and the distance between correction point and the corresponding measuring point, the accuracy of land survey has enhanced by more than $75 \%$. It is obvious that after being corrected by precision correction model, accuracy of land survey data can be enhanced largely, with the accuracy being close to physical truth. 


\begin{tabular}{|l|l|l|l|l|l|c|c|}
\hline \multicolumn{2}{|c|}{ Plotting coordinates } & \multicolumn{2}{c|}{ correction coordinates } & Measurement coordinates & \multicolumn{2}{c|}{ distance } \\
\hline \multicolumn{1}{|c|}{$\mathbf{x}$} & $\mathbf{y}$ & $\mathbf{x}$ & $\mathbf{y}$ & $\mathbf{x}$ & $\mathbf{y}$ & $\mathbf{D}_{\mathbf{1}}$ & $\mathbf{D}_{\mathbf{2}}$ \\
\hline 457987.241 & 2612998.051 & 457986.781 & 2612998.671 & 457985.915 & 2612999.014 & 1.639 & 0.931 \\
\hline 457721.974 & 2612870.809 & 457722.448 & 2612869.906 & 457723.183 & 2612870.270 & 1.324 & 0.820 \\
\hline 458103.7 & 2612732.784 & 458102.840 & 2612731.843 & 458103.113 & 2612732.037 & 0.950 & 0.335 \\
\hline 458517.776 & 2613218.029 & 458518.328 & 2613217.891 & 458519.002 & 2613218.104 & 1.228 & 0.707 \\
\hline 458491.896 & 2613127.45 & 458492.368 & 2613128.157 & 458492.012 & 2613127.879 & 0.444 & 0.452 \\
\hline 458435.823 & 2613034.714 & 458436.233 & 2613035.114 & 458435.848 & 2613034.816 & 0.105 & 0.487 \\
\hline 458677.367 & 2613000.208 & 458676.913 & 2613001.203 & 458676.038 & 2613002.137 & 2.342 & 1.280 \\
\hline 458688.15 & 2613108.04 & 458687.951 & 2613109.102 & 458688.510 & 2613109.829 & 1.825 & 0.917 \\
\hline
\end{tabular}

Table2: Some of the nodes coordinate

notes: (1) The D1 in table means the distance between plotting point and the corresponding measuring point.

(2) The D2 in table means the distance between correction point and the corresponding measuring point.

\section{CONCLUSION}

The results of the experiment show that the technology for precision correction of land survey data is useful to correct the errors of plotting coordinates of the land-use feature nodes. It can achieve the purpose of making the accuracy of plotted data and measurement data basically close, especially in the flat area, where most of the corrected coordinates is close to the measurement, while in region of larger relief, the effects of the accuracy correction are unstable, some of the errors of coordinates become larger. In order to improve the technology's adaptability to different area, there could be more in-depth study on accuracy correction model and refinement of computational area based on the results of this paper.

\section{REFERENCES}

[1] Zhang Jiang-xue. Research on Data Quality Control in The Second Rural Land Investigation[D]. 2010.

[2] J Feng. Application of 3S Technology to the Updating Survey of Land. International Conference on Environmental Science and Information Application Technology (ESIAT), 2009; 1:604-607.
[3]Xie Zhi-ru. Ortho-rectification on SPOT5 Satellite Remote Sensing Image in Region of Relief[C]. The Application of New Technology in Land Survey and the Development of Land Science Technology, 2005:318-321.

[4]Zhao Jian-hui. On the Data Processing Methods of QuickBird Image in Land Resources Updating Investigation[J]. Bulletin of Surveying and Mapping,2011,(7):40-63.

[5] G. B. GROOM, R. M. FULLER, A. R JONES .Contextual correction: techniques for improving land cover mapping from remotely sensed images.International Journal of Remote Sensing,Vol. 17, Iss. 1, 1996.

[6] N.G. Kardoulas, A.C. Bird, and A.I. Lawan. Geometric Correction of SPOT and Landsat Imagery: A Comparison of Map and GPS-Derived Control Points. Photogrammetric Engineering \& Remote Sensing, Vol. 62, No. 10, October 1996, pp. 1173-1177.

[7] A. J. De Leeuw, L. M. M. Veugen, H. T. C. Van Stokkom .Geometric correction of remotely-sensed imagery usiing ground control points and orthogonal 
polynomials.International Journal of Remote

Sensing,Vol. 9, Iss. 10-11, 1988.

[8] Manadili Y., Novak K. 1996, Precision

Rectification Of SPOT Imagery Using The

Direct Linear Transformation

Model,Photogrammetric Engineering And

Remote Sensing, Vol. 62,No. 1, pp 67-72.

[9]Xu Yu-ying, Xu Jian-gang, Ji Guang-Sen. The

Quality Control of Digital Industry in Land Use UpdateSurvey[C]. Proceedings about E-Government and Geographic InformationTechnology, 2005:137-138,143.

[10] Xu Hai-Jun. Study of Quality Control Based on the Second Land Survey.A Dissertation Submitted for the Degree of Master.2009.

[11] Su Hao-jiang, Li De-ming. The Precision Control of Inside-to-outside Procedure in Large Scale Digital Aerophotogrammetry Mapping. Modern Surveying and Mapping. Vol.25 No 4 Aug. 2005: 19-21.

[12] Fan Ming-hua, Yang Luo-jun, Xu Hong-xin. Processing Method of Debris in Farmland on the Map. Modern Surveying and Mapping. Vol.33,No.6 Nov.2010.

[13]LI De-ren Wang Bao-gen. An introduction Photogrammetry and remote sensing[M]. Surveying and Mapping Press 2006 